\title{
Polymer sphere lithography for solid oxide fuel cells: a route to functional, well-defined electrode structures
}

\author{
Evan C. Brown, ${ }^{a}$ Stephen K. Wilke, ${ }^{a}$ David A. Boyd, ${ }^{b}$ David G. Goodwin ${ }^{b}$ and Sossina M. Haile ${ }^{* a}$ \\ Received 9th October 2009, Accepted 4th December 2009 \\ First published as an Advance Article on the web 25th January 2010 \\ DOI: 10.1039/b920973e
}

\begin{abstract}
As a first step towards mechanistic studies of fuel cell electrodes with both well-defined and functionally representative structural features, two-dimensional anti-dot metal films with tunable features are prepared. The fabrication employs a facile, sacrificial templating method, known as polymer sphere lithography, and the resulting metal films are fully connected, yet fully porous. Using initial bead sizes in the range of $500 \mathrm{~nm}$ to $3.2 \mu \mathrm{m}$ and oxygen plasma etching to remove from $1 / 4$ to $3 / 4$ of the original bead diameter, computed triple phase boundary densities in the porous films of 2,000 to $43,500 \mathrm{~cm} \mathrm{~cm}^{-2}$ are achieved. Image analysis shows the computed (theoretical) and experimental structural features to be in good agreement, demonstrating sufficient perfection in the films for electrochemical studies.

Furthermore, thermal stability under hydrogen of thermally evaporated Ni films is excellent, with negligible change in triple phase boundary length as required for quantitative electrochemical measurements. Ultimately, these two-dimensional metallic networks may also serve as the platform for future fabrication of three-dimensional electrodes with truly optimized structural features.
\end{abstract}

\section{Introduction}

Dramatic breakthroughs in the materials, particularly electrode materials, for solid oxide fuel cells (SOFCs) have been reported in recent years. ${ }^{1-3}$ Fundamental understanding of the electrocatalytic pathways, however, has not kept pace, and consequently trial and error continues to be the primary mode of SOFC component development. Despite the lack of detailed mechanistic insight, it is generally understood that the triple phase boundaries (TPBs) of SOFC electrodes, at which gas, electrode and electrolyte phases are simultaneously in contact, and hence all reactant and product species (ions, electrons, neutral molecules) are simultaneously present, serve as the predominant sites for electrochemical reactions. It is also recognized that the electrodes must further serve the triple functions of electron transport, ion transport and gas phase transport.

Given these multiple functional requirements, fuel cell electrodes are typically composite structures formed of an electrolyte (ion conductor), metal (electron conductor), although a single component may transport ions and electrons, and a fugitive pore former. Additional catalysts may also be introduced, but this is generally not required at the high temperatures of SOFCs. For ease of large-scale manufacturing, such composites are most often prepared using traditional ceramics processing methods. While technologically useful, such fabrication approaches yield ill-defined structures that preclude deconvolution of microstructural and compositional influences on electrochemical reaction rates and ultimately prevent elucidation of reaction

${ }^{a}$ Department of Materials Science, California Institute of Technology, Pasadena, CA, 91125, USA. E-mail: smhaile@caltech.edu

${ }^{b}$ Department of Mechanical Engineering, California Institute of Technology, Pasadena, CA, 91125, USA mechanisms. ${ }^{4}$ In recognition of the need to examine geometrically well-defined structures, some recent mechanistic studies have employed two-dimensional electrodes patterned onto the electrolyte of interest..$^{5-8}$ This approach has begun to bear fruit and mechanistic models have begun to be developed;9,10 however, challenges in understanding 'real' fuel cells remain because the two-dimensional patterns have a substantially lower areal density of TPBs (defined as the TPB length per unit of projected electrolyte area) than the systems they represent. Specifically, conventional photolithographic techniques with a minimum feature size of about $5 \mu \mathrm{m}$ can attain a maximum areal TPB density of $2,000 \mathrm{~cm} \mathrm{~cm}^{-2} .{ }^{5}$ In contrast, typical fuel cell electrodes boast values as high as $800,000 \mathrm{~cm} \mathrm{~cm}^{-2}$. ${ }^{4}$ Such significant microstructural differences can plausibly induce differences in reaction pathways. Accordingly, there is a pressing need to obtain geometrically defined electrode structures with tunable feature sizes that are more relevant to SOFC electrocatalysis.

We demonstrate here a facile fabrication strategy that permits access to TPB areal densities over an enormous range (2,000 to $43,500 \mathrm{~cm} \mathrm{~cm}^{-2}$ ) in which monodisperse polymer spheres serve as sacrificial templates (Fig. 1). After deposition of a single polymer layer, the beads are radially etched in an oxygen plasma, so as to create vias between them. Metal is deposited using a line-of-sight deposition method that enables the still-round PS to serve as a lithographic mask. Upon removal of the polymer template, the desired porous structure is obtained.

Polymer sphere lithography and, in particular, nanosphere lithography have recently gained attention for a wide range of applications ranging from novel nanofabrication techniques and photonic crystals to superhydrophobic surfaces. ${ }^{11-13}$ Most often, the film deposition is limited to isolated locations corresponding to the interstices between the template beads. ${ }^{14-16}$ With control of the fabrication process, however, the film may form a fully interconnected, yet fully porous network, acquiring what has 
(a)
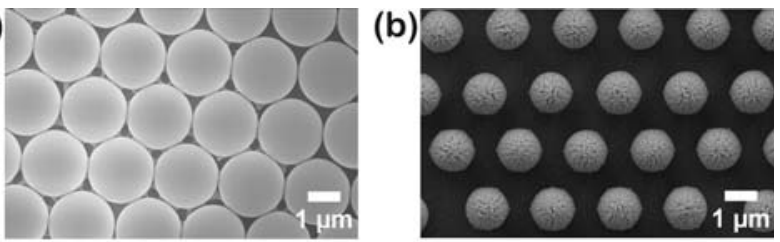

(c)

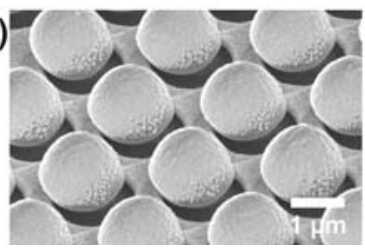

(d)

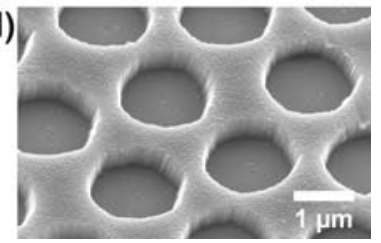

Fig. 1 The polymer lithography process, all on yttria-stabilized zirconia (YSZ): (a) monolayer of $500 \mathrm{~nm}$ polystyrene (PS) beads; (b) diameter of beads reduced via oxygen plasma etching; (c) metal $(\mathrm{Au})$ deposited by thermal evaporation; (d) PS beads removed.

been termed an 'anti-dot' configuration. ${ }^{17,18}$ Though not previously explored in the fuel cell context, the anti-dot structure is ideal for advancing fundamental studies of electrocatalytic pathways in fuel cell electrodes. The periodicity provided by polymer bead self-assembly is not critically important for such studies; however, sufficient knowledge of microstructural parameters is required, as is high temperature stability. Accordingly, both factors are evaluated here.

\section{Results and discussion}

\subsection{Fabrication approaches}

Achieving a single layer of the polymer beads across the entirety of the substrate is essential to the success of the polymer sphere lithographic technique. Several approaches for monolayer deposition have been pursued in the literature, with varying degrees of complexity and experimental constraints. The most common methods are combined sedimentation plus evaporation, ${ }^{19,20}$ spin-coating plus evaporation, ${ }^{21}$ and controlled evaporation in combination with gradual substrate withdrawal from the solution (dip-coating). ${ }^{22}$ More exotic methods include electrophoretic assembly (suitable only to conducting substrates), and high pressure infusion in combination with ultrasonication. ${ }^{23-25}$ While sedimentation, dip-coating and spincoating are relatively straightforward methods that produce structures with regularity sufficient for electrocatalysis studies, they suffer from the tendency of the processes to yield regions with multiple layers and others entirely devoid of the polymer spheres. Furthermore, achieving adequate control of the evaporation step for the former two can require excessive processing times.

Here we employ spin-coating as a facile means of obtaining the desired monolayers on SOFC electrolyte materials, yttria-stabilized zirconia (YSZ) and samaria-doped ceria (SDC), where a slight variation of the standard spinning approach is necessary for larger $(>1 \mu \mathrm{m})$ beads. Utilization of electronically insulating substrates precludes electrodeposition as a means of subsequent growth of the metallic film, motivating the two-step process pursued here of bead etching and vapor phase metal deposition.

(a)

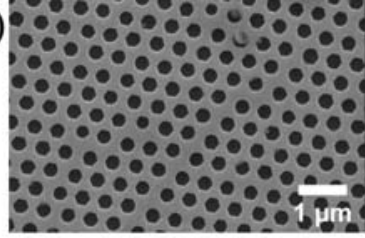

(b)

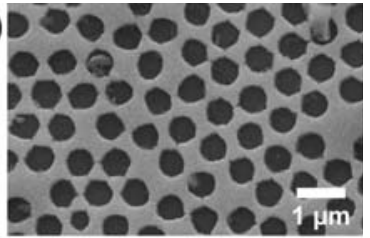

(c)

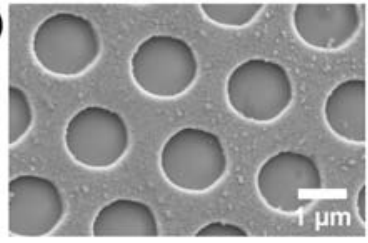

(d)

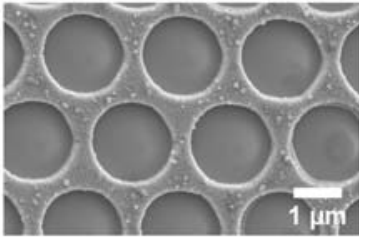

(e)

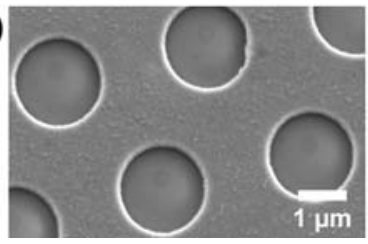

(f)

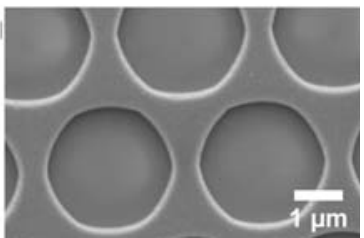

Fig. 2 Selection of representative copper anti-dot metal films on YSZ showing range of feature sizes achieved using polymer sphere lithography: (a) $500 \mathrm{~nm}$ initial bead size; (b) $790 \mathrm{~nm}$ initial bead size; (c) $2 \mu \mathrm{m}$ initial bead size, heavily etched; (d) $2 \mu \mathrm{m}$ initial bead size, lightly etched; (e) $3.2 \mu \mathrm{m}$ bead size, heavily etched; (f) $3.2 \mu \mathrm{m}$ initial bead size, lightly etched.

For the plasma-etched spheres to serve as effective templates, it is necessary for film growth to be limited to line-of-sight methods that avoid deposition in the void space on the underside of the round beads. Thermal evaporation has been employed in this work for conventional metals (copper, nickel, titanium, titanium/ gold, aluminium), whereas electron-beam evaporation has been used for Pt (due to its high melt temperature), with equal effectiveness in all cases. A representative selection of the types of anti-dot electrode structures obtained in this work is presented in Fig. 2. The ability to fabricate anti-dot structures from a range of metals on multiple electrolyte materials is essential for ultimate fundamental electrochemical studies.

Nanosphere lithography. Monolayers of as-purchased, carboxyl-functionalized polystyrene (PS) beads with diameters less than $1 \mu \mathrm{m}$ (specifically, 500, 680 and $790 \mathrm{~nm}$ ) were prepared by spin-coating onto a hydrophilic surface (Fig. 3), where the optimal spin acceleration, ultimate spin rate and dwell time were each found to depend on the bead diameter. Non-functionalized beads displayed insufficient attraction to one another and, consequently, spin-coating resulted in large areas devoid of the template, despite exhaustive attempts at optimizing the spinning conditions. The next step, etching of polystyrene by oxygen plasma treatment, is well-known and was readily applied here. ${ }^{18}$ We observed that short treatments generate contacts between the beads, presumably as a consequence of softening of the polymer. This undesirable 'necking' was avoided by longer treatments that remove at least $1 / 4$ of the original bead diameter. Finally, in order to ensure the removal of the template without damage to the desired pattern, the film thickness is limited to approximately $1 / 2$ the diameter of the etched beads, with the further constraint that a minimum thickness of about $150 \mathrm{~nm}$ is required in order to 
attain acceptable electron transport properties in the porous film. These considerations preclude fabrication of useful anti-dot electrodes with PS beads of less than $500 \mathrm{~nm}$ in diameter.

(a)

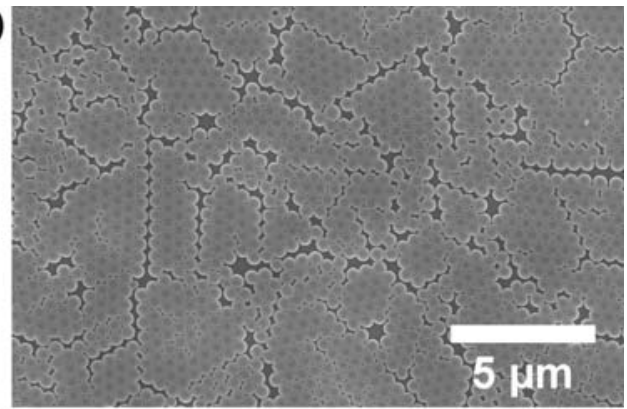

(b)

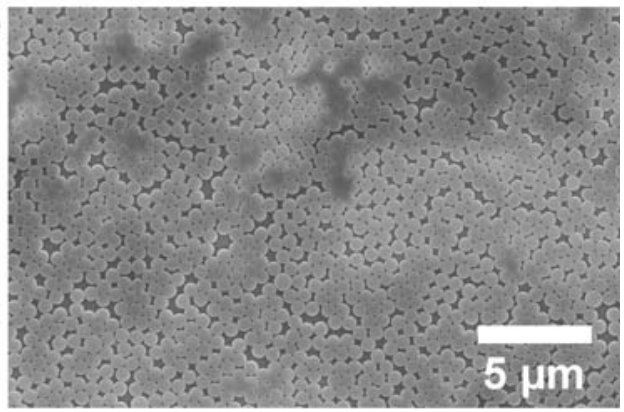

(c)

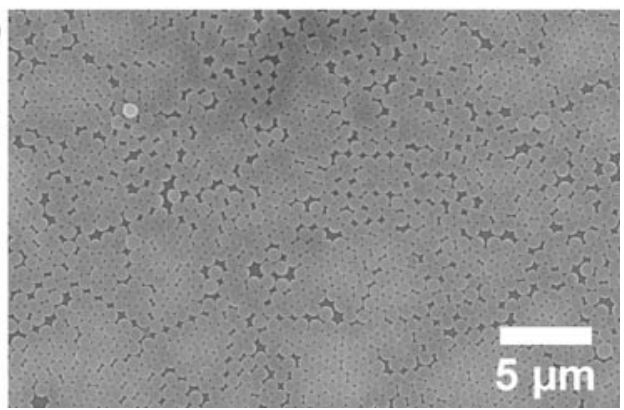

Fig. 3 Substrate coverage via a single spin-coat for polystyrene nanospheres on YSZ: (a) $500 \mathrm{~nm}$ diameter; (b) $680 \mathrm{~nm}$ diameter; and (c) 790 $\mathrm{nm}$ diameter. Each image contains $\sim 1200$ beads.
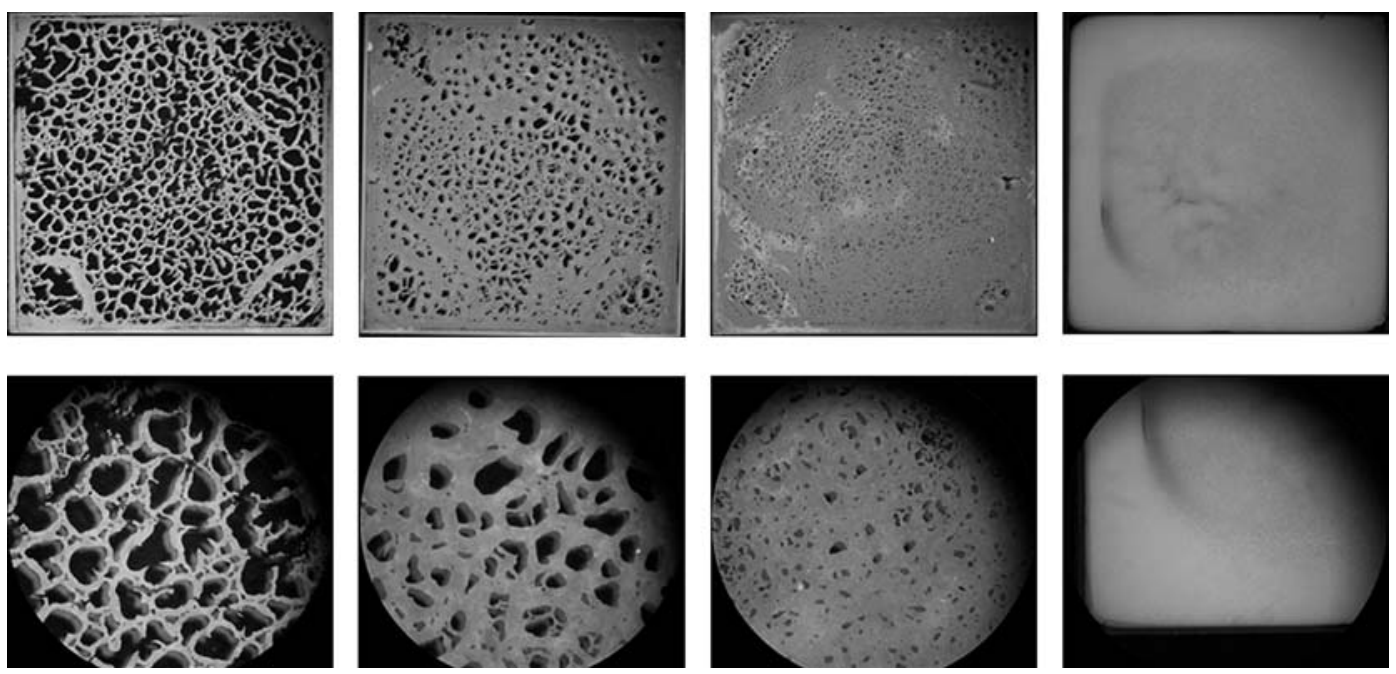

Fig. 4 Sequential spin coats with water-washing in-between steps. $2 \mu \mathrm{m}$ beads shown here on YSZ. Substrates are $1 \mathrm{~cm} \times 1 \mathrm{~cm}$. 
Table 1 Comparison of theoretical and experimental TPB length areal density and percent electrolyte exposure for different initial PS bead diameters

\begin{tabular}{|c|c|c|c|c|c|c|c|}
\hline $\begin{array}{l}\text { Initial bead } \\
\text { diameter, } \varphi_{\mathrm{i}} / \mu \mathrm{m}\end{array}$ & $\begin{array}{l}\text { Final pore } \\
\text { diameter, } \\
\varphi_{\mathrm{f}} / \mu \mathrm{m}\end{array}$ & $\begin{array}{l}\text { Pore diameter } \\
\text { Gaussian } \\
\text { width } / \mu \mathrm{m}\end{array}$ & $\begin{array}{l}\text { Theoretical } \\
\text { TPB length } \\
\text { density } / \mathrm{m} \mathrm{cm}^{-2}\end{array}$ & $\begin{array}{l}\text { Experimental } \\
\text { TPB length } \\
\text { density } / \mathrm{m} \mathrm{cm}^{-2}\end{array}$ & $\begin{array}{l}\text { Theoretical } \\
\text { percent } \\
\text { electrolyte } \\
\text { exposure }\end{array}$ & $\begin{array}{l}\text { Experimental } \\
\text { percent electrolyte } \\
\text { exposure }\end{array}$ & $\begin{array}{l}\text { Electrolyte } \\
\text { exposure } \\
\text { standard } \\
\text { deviation }\end{array}$ \\
\hline 0.5 & 0.3 & 0.06 & 435 & 406 & $32.6 \%$ & $31.5 \%$ & 12.02 \\
\hline 2 & 1.29 & 0.07 & 117 & 112.8 & $37.7 \%$ & $33.4 \%$ & 6.70 \\
\hline 2 & 1.58 & 0.08 & 143 & 137.3 & $56.6 \%$ & $49.7 \%$ & 4.69 \\
\hline 3.2 & 1.72 & 0.10 & 61 & 57.6 & $26.2 \%$ & $23.5 \%$ & 3.60 \\
\hline
\end{tabular}

which the first deposition has yielded a mixture of void regions, monolayer regions and multilayer regions is gently rinsed with water to remove the excess layers in the multilayer regions and the spin-coating is repeated to induce deposition in the void regions. The process is repeated multiple times until the void regions constitute less than about $10 \%$ of the substrate area, beyond which multilayer regions cannot be removed by a gentle rinse with water. In a final step, a small amount of the PS suspension is directly applied to the substrate and allowed to dry, eliminating the remaining void regions. By this method, it was possible to prepare comprehensive monolayers of PS beads up to $3.2 \mu \mathrm{m}$ in diameter (Fig. 5). The subsequent plasma treatment, metal deposition and template removal steps then proceed as described for the nanosphere lithography, where, again, a minimum of $1 / 4$ of the bead diameter must be removed in order to prevent necking during oxygen plasma treatment.

\subsection{Microstructural features}

Given the importance of triple-phase boundaries for SOFC electrocatalysis, the TPB areal density (defined per unit of projected electrolyte area as already described above) is a key parameter describing the microstructural features of these or any fuel cell electrode. A further important parameter in the case of the two-dimensional electrodes prepared here is the metal coverage, or inversely, the fraction of exposed electrolyte area. With knowledge of these two parameters and an ability to tune them over a wide range, it becomes possible to achieve the goal of deconvoluting microstructural and compositional influences on electrocatalysis rates.

For a perfect micro/nanosphere lithographic process in which the template beads display ideal periodicity over the entirety of the substrate, both the TPB areal density, $\rho_{\mathrm{TPB}}$, and the electrolyte exposure, $f_{\text {lyte }}$, can be computed from knowledge of the starting bead size and the extent of size reduction induced upon plasma etching. The theoretical values of these two quantities are given in eqn (1) and 2, respectively, as functions of the initial $\left(\phi_{i}\right)$ and final $\left(\phi_{f}\right)$ diameters of the PS beads.

$$
\begin{aligned}
& \rho_{T P B}^{\text {theo }}=\frac{2 \pi}{\sqrt{3}}\left(\frac{\phi_{f}}{\phi_{i}}\right) \frac{1}{\phi_{i}} \\
& f_{\text {lyte }}^{\text {theo }}=\frac{\pi}{2 \sqrt{3}}\left(\frac{\phi_{f}}{\phi_{i}}\right)^{2}
\end{aligned}
$$

For an imperfect fabrication process, many kinds of defects exist: disordered regions of PS beads, multilayer and void areas
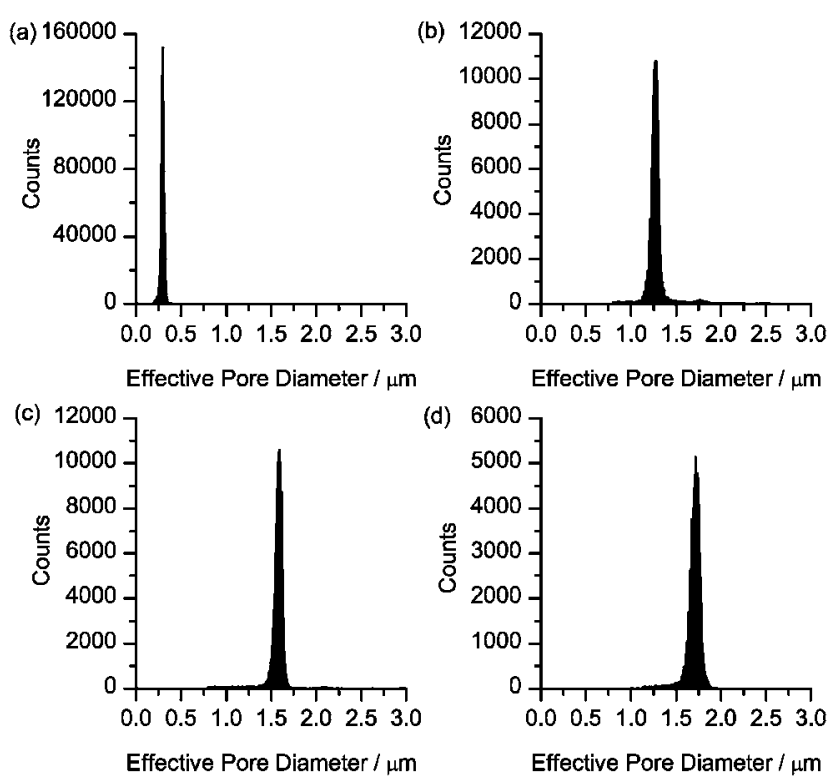

Fig. 6 Pore diameter histograms of copper networks on YSZ, reflecting the different starting PS bead sizes: (a) $500 \mathrm{~nm}$ etched to $300 \mathrm{~nm}$; (b) $2 \mu \mathrm{m}$ etched to $1.29 \mu \mathrm{m}$; (c) $2 \mu \mathrm{m}$ etched to $1.58 \mu \mathrm{m}$; (d) $3.2 \mu \mathrm{m}$ etched to $1.72 \mu \mathrm{m}$.

(areal defects); grain boundaries between ordered regions (line defects); and single missing PS beads within an ordered region (point defects). To assess the influence of these random structural elements, a continuous string of scanning electron microscopy (SEM) images (typically numbering from 100-400 images per sample) was collected from border to border for four representative films. From each image the following parameters were determined: the number and diameter of the pores, the metalsubstrate interface (i.e. TPB) length, and the exposed electrolyte area fraction. The film characteristics and measured results are summarized in Table 1 and Fig. 6 and 7.

The distribution of pore diameters (Fig. 6) in the films was found to be rather narrow, with a Gaussian peak width that is $\sim 2 \%$ of the mean diameter. This distribution largely reflects the size distribution in the as-purchased PS beads, also about $2 \%$, as oxygen plasma treatment was observed to remove material from the beads in a spatially uniform fashion, both radially and from bead to bead (Fig. 1b). ${ }^{26}$ In addition to the pores represented in the histograms of Fig. 6, a small number of pores (less than 1\% of the total) with large diameters, $>3 \mu \mathrm{m}$, was also observed. These are taken to reflect regions in which multilayers of PS beads occurred. The contribution of these large diameter pores to the overall $\rho_{\mathrm{TPB}}$ is small, as indicated by the good agreement between 

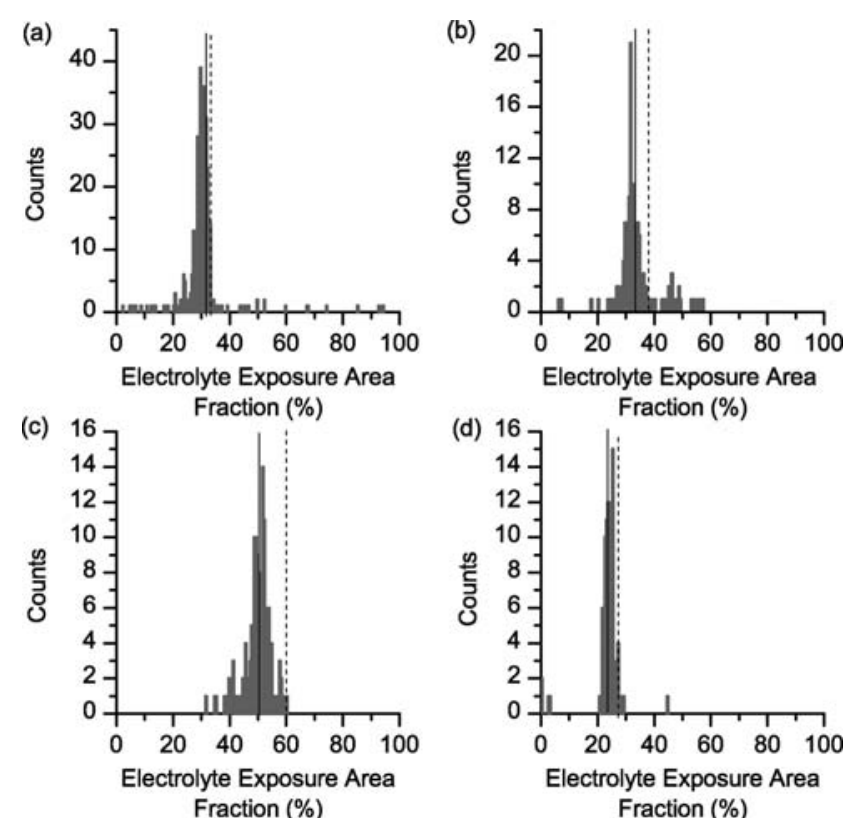

Fig. 7 Electrolyte exposure area fraction histograms of copper networks on YSZ, reflecting different starting PS bead sizes: (a) $500 \mathrm{~nm}$ etched to $300 \mathrm{~nm}$; (b) $2 \mu \mathrm{m}$ etched to $1.29 \mu \mathrm{m}$; (c) $2 \mu \mathrm{m}$ etched to $1.58 \mu \mathrm{m}$; (d) $3.2 \mu \mathrm{m}$ etched to $1.72 \mu \mathrm{m}$. Solid and dashed lines indicate the average and theoretical values, respectively.

the theoretical and experimental values of this parameter, Table 1, and they are omitted from the plotted range for clarity.

The image-to-image variation in the exposed electrolyte area (Fig. 7) shows that the variability in $f_{\text {lyte }}$ is more significant than the pore diameter variability. The widest distribution in $f_{\text {lyte }}$ is evident for the film prepared using $500 \mathrm{~nm}$ PS beads, where the standard deviation is $12 \%$ of the substrate area (i.e. $f_{\text {lyte }}$ is $31.5 \pm$ $12.0 \%$ ). Moreover, in all cases, the observed exposed electrolyte area was lower than that computed from the initial and final PS bead sizes. This can be attributed to the occurrence of point and line defects in the PS 2-D crystals, as well as the presence of disordered regions in the monolayer. The statistics surrounding the two films prepared using PS beads with an initial diameter of $2 \mu \mathrm{m}$ suggest that line and point defects become increasingly important as the extent of etching is minimized. In the case of the film obtained from lightly etched PS beads $\left(\phi_{f}=1.58 \mu \mathrm{m}\right)$ there is a large difference between $f_{\text {lyte }}^{\text {theo }}$ and $f_{\text {lyte }}^{\text {xxp }}(56.6 v s .49 .7 \%)$. When the beads are more heavily etched $\left(\phi_{f}=1.29 \mu \mathrm{m}\right)$, the difference decreases, whereas the difference between $p_{\mathrm{TPB}}^{\text {theo }}$ and $p_{\mathrm{TP}}^{\mathrm{exp}}$ and the distribution of pore sizes for the two films are essentially the same. The behavior can be understood as follows. In the case of the lightly etched film, isolated missing beads (both point defects and dislocations behave as isolated, absent beads in a 2-D crystal) become a significant portion of the open area available for metal deposition, and, in this manner, such defects increasing in number dominate the coverage features.

The histograms of exposed electrolyte area display significant numbers of occurrences outside of what is roughly the main peak. As already indicated, regions with exposed electrolyte area below the mean occur as a consequence of defects in the 2-D crystals (i.e. voids in the PS bead array), whereas regions with higher fractions of exposed electrolyte area occur because multi- layers form during the PS bead deposition process (i.e. excessive coverage of the substrate with PS beads). The $500 \mathrm{~nm}$ diameter nanospheres generate films in which slightly less than $10 \%$ of the regions display significant PS bead void areas, whereas $4 \%$ display multi-layered areas. In contrast, for both sizes of microspheres ( 2 and $3.2 \mu \mathrm{m}$ ) the regions affected by voids in the PS bead array are less than 5\%, indicating that the multiple deposition process has more comprehensively filled the monolayer. The occurrence of multilayer regions for the $2 \mu \mathrm{m}$ microspheres accounts for $11 \%$ of the regions imaged, whereas for the $3.2 \mu \mathrm{m}$ it is only $1 \%$, suggesting that multi-layer removal becomes facile as the bead size increases.

Overall, despite the imperfection of the monolayer deposition process, the theoretical and experimental values, respectively, of $f_{\text {lyte }}$ and of $\rho_{\text {TPB }}$ agree quite well with one another, indicating that the fabrication is, in fact, rather controlled. Indeed, all of the experimental $\rho_{\text {TPB }}$ values are within $93 \%$ of the theoretical, and this was found to hold irrespective of substrate employed or metal deposited. Accordingly, the geometric features of any sample prepared by the methodology presented here can, within a reasonable degree of certainty, be predicted from knowledge of $\phi_{i}$ and $\phi_{f}$.
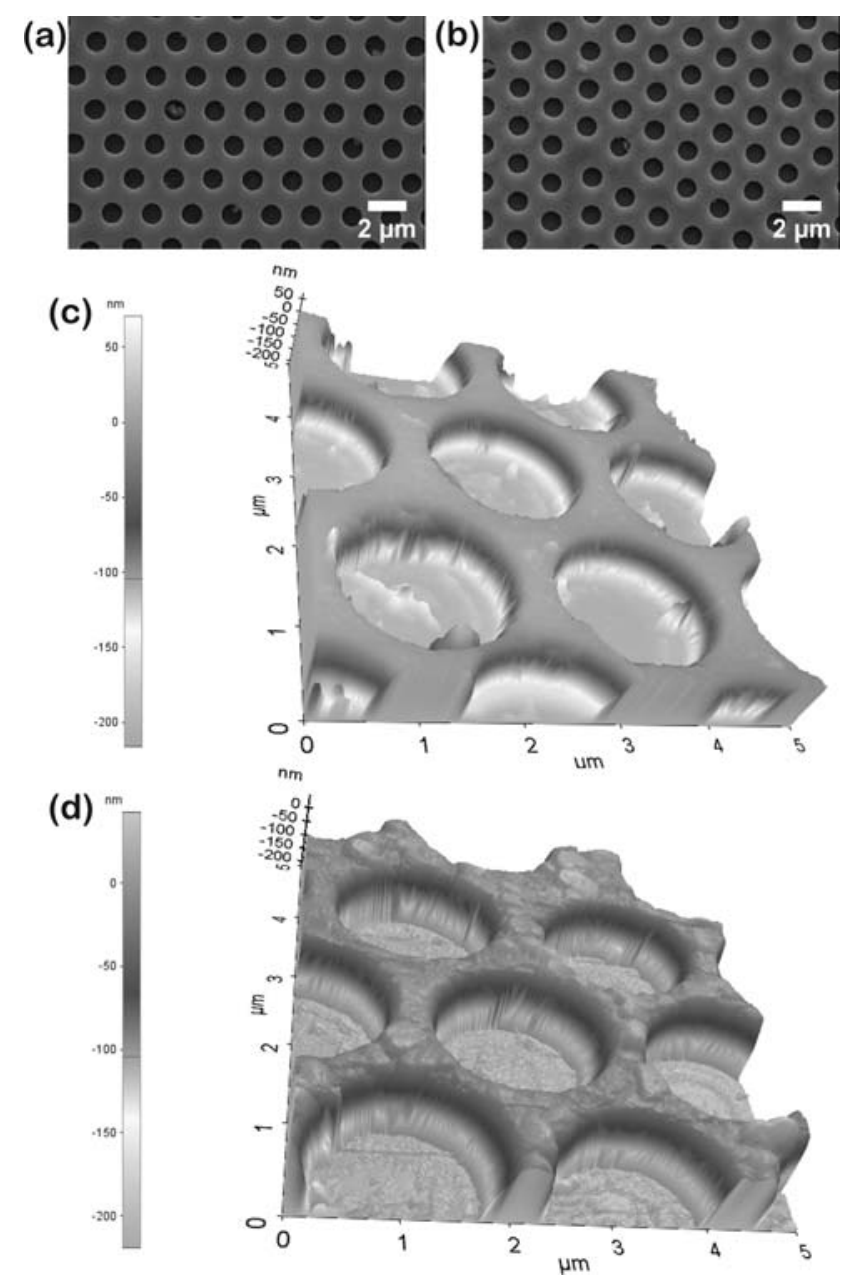

Fig. 8 Images of anti-dot porous nickel network (a) and (c) before thermal treatment at $600^{\circ} \mathrm{C}$ under hydrogen $\left(\mathrm{pH}_{2}=0.1 \mathrm{~atm}\right)$; and (b) and (d) after treatment. (a) and (b) are top-down SEM images; (c) and (d) are AFM images. 


\subsection{Thermal stability}

An additional important characteristic of model fuel cell electrodes is thermal stability. That is, the TPB length and exposed electrolyte area must not change during the course of a hightemperature electrochemical measurement. To evaluate thermal stability, nickel metal networks were annealed at $600{ }^{\circ} \mathrm{C}$ for over $48 \mathrm{~h}$ under humidified hydrogen, typical operating conditions for the anode of an intermediate temperature SOFC. SEM images reveal no discernable microstructural evolution as a consequence of the heat treatment (Fig. 8ab), whereas slight changes are visible in the atomic force microscopy (AFM) images (Fig. 8cd). Specifically, the nickel surface roughens, from a root mean square (rms) roughness of approximately 8 to $14 \mathrm{~nm}$, and the grains undergo slight growth, in a direction limited largely to the surface normal. No other metal networks were subjected to high temperatures.

\section{Experimental details}

\section{Substrate preparation}

The YSZ substrates (MTI Corporation) used are (100) singlecrystals, and the samaria-doped ceria substrates are epitaxially deposited thin films on single-crystal YSZ via pulsed laser deposition. ${ }^{27}$ For subsequent use for nanosphere deposition, the substrates were exposed to an oxygen plasma for 5 min at $75 \mathrm{~W}$ and 250 mTorr (Technics Planar Etch II) to enhance hydrophilicity. In contrast, as-purchased or as-fabricated substrates were directly used for microsphere deposition.

\section{Nanosphere deposition}

Monolayers were spun using a Laurell, WS-400B-6NPP/LITE, with a $10 \mathrm{wt} \%$ suspension of PS nanospheres, functionalized with carboxyl groups (Bangs Laboratories, Inc. ${ }^{\mathrm{TM}}$ ). Before spin coating on the substrate, the as-received PS suspension is sonicated to ensure the beads are homogeneously dispersed. Exactly $35 \mu \mathrm{L}$ of the suspension was manually spread over the entire $1 \mathrm{~cm}$ $\times 1 \mathrm{~cm}$ substrate before spinning. The final RPM of the spin coater was 3000 RPM, with varying accelerations depending on the starting PS diameter. The PS monolayer was radially etched in the same oxygen plasma system, but at elevated powers $(75-200 \mathrm{~W})$. In this step, the beads do not move from their original positions. Typical etching times were anywhere from 5 to $20 \mathrm{~min}$.

\section{Microsphere deposition}

Non-functionalized PS beads were used for the larger diameters (Thermo Scientific), as $10 \mathrm{wt} \% .35 \mu \mathrm{L}$ of the PS suspension was manually spread over the entire substrate and spun as before. The spin coater was spun at 4000 RPM. A standard laboratory spray bottle was used to employ the water-wash method described previously. If the spin-wash-dry cycle was repeated too many times, immovable multilayers would form. For the $2 \mu \mathrm{m}$ spheres, the cycle was repeated 3 times; for the $3.2 \mu \mathrm{m}$ spheres, the cycle was repeated 6 times.

\section{Metal deposition}

An in-house constructed thermal evaporation system was used to deposit copper, nickel, titanium, titanium/gold, or aluminium (Alfa Aesar, 99.98+\%) at $10^{-5}$ Torr. Platinum networks were evaporated using an electron beam evaporator (re-manufactured CHA MK-40). The now covered PS beads were removed with an acetone-soaked cotton swab; regardless of the metal deposited, the surface became lustrous after wiping repeatedly, indicating the PS was gone.

\section{Microstructure analysis}

Optical photos were taken using a Nikon SMZ1500 stereomicroscope. Electron micrographs were taken on an LEO 1550VP Field Emission SEM. Atomic force microscopy (AFM) images were collected using a Park Systems XE-70 AFM. Image analyses were performed using ImageJ 1.410 freeware. Statistical data from the series of SEM photos captured a little over $1 \%$ of the total substrate area, constituting 100-400 photos, depending on the magnification used. $60,000-650,000$ pores per sample were evaluated: the pore areas were assumed to be perfectly circular, and the diameters were calculated from the individual pore areas.

\section{High temperature stability}

$200 \mathrm{~nm}$ thick nickel networks were brought to $600{ }^{\circ} \mathrm{C}$ under flowing $98.7 \% \mathrm{H}_{2}$ and $1.3 \% \mathrm{H}_{2} \mathrm{O}$ and held there for $50 \mathrm{~h}$.

\section{Conclusions}

Polymer sphere lithography has been utilized to create twodimensional metallic networks on fuel cell electrolyte materials. Although the fabrication process involves somewhat imprecise, random elements, the experimental variation from the expected geometries is extremely small. Under fuel cell operating conditions, the structures, and, hence, the TPB and exposed electrolyte area fraction values, exhibit remarkable high temperature stability. These well-defined and well-behaved electrode structures access a wide range of TPB regimes, lending themselves to future mechanistic studies on electrolyte-electrode material systems, as well as providing a strong experimentally correlated basis for computational modeling. Beyond mechanistic studies, these anti-dot structures may ultimately serve as platforms for fabrication of three-dimensional electrodes with truly optimized architectures.

\section{Acknowledgements}

The authors gratefully acknowledge William Chueh for valuable discussions and for providing epitaxial ceria, as well Chang-yi $\mathrm{Li}$ for providing AFM images. This work is supported by the Global Climate and Energy Program (GCEP) of Stanford University, funded by a consortium of industrial sponsors.

\section{Notes and references}

1 A. Atkinson, S. Barnett, R. J. Gorte, J. T. S. Irvine, A. J. McEvoy, M. Mogensen, S. C. Singhal and J. Vohs, Nat. Mater., 2004, 3, 17-27.

2 S. M. Haile, Acta Mater., 2003, 51, 5981-6000.

3 Z. Shao and S. M. Haile, Nature, 2004, 431, 170-173. 
4 J. R. Wilson, W. Kobsiriphat, R. Mendoza, H. Chen, J. M. Hiller, D. J. Miller, K. Thornton, P. W. Voorhees, S. B. Adler and S. A. Barnett, Nat. Mater., 2006, 5, 541-544.

5 J. Mizusaki, H. Tagawa, T. Saito, K. Kamitani, T. Yamamura, K. Hirano, S. Ehara, T. Takagi, T. Hikita, M. Ippommatsu, S. Nakagawa and K. Hashimoto, J. Electrochem. Soc., 1994, 141 2129-2134.

6 A. Bieberle, L. P. Meier and L. J. Gauckler, J. Electrochem. Soc., 2001, 148, A646-A656.

7 J. Fleig, Annu. Rev. Mater. Res., 2003, 33, 361-382.

8 F. S. Baumann, J. Fleig, G. Cristiani, B. Stuhlhofer, H.-U. Habermeier and J. Maier, J. Electrochem. Soc., 2007, 154, B931-B941.

9 A. Bieberle and L. J. Gauckler, Solid State Ionics, 2002, 146, 23-41.

10 D. G. Goodwin, H. Zhu, A. M. Colclasure and R. J. Kee, J. Electrochem. Soc., 2009, 156, B1004-B1021.

11 C. L. Haynes and R. P. Van Duyne, J. Phys. Chem. B, 2001, 105, 5599-5611.

12 S. Mahajan, M. Abdelsalam, Y. Suguwara, S. Cintra, A. Russell, J. Baumberg and P. Bartlett, Phys. Chem. Chem. Phys., 2007, 9, 104-109.

13 J. Shiu, C. Kuo, P. Chen and C. Mou, Chem. Mater., 2004, 16, 561564.

14 J. Boneberg, F. Burmeister, C. Schafle and P. Leiderer, Langmuir, 1997, 13, 7080-7084.
15 J. Hulteen and R. P. Van Duyne, J. Vac. Sci. Technol., A, 1995, 13, $1553-1558$

16 F. Burmeister, C. Schafle, T. Matthes, M. Bohmisch, J. Boneberg and P. Leiderer, Langmuir, 1997, 13, 2983-2987.

17 E. Spada, A. Da Rocha, E. Jasinski, G. Pereira, L. Chavero, A. Oliveira, A. Azevedo and M. Sartorelli, J. Appl. Phys., 2008, 103, 114306-5.

18 C. Haginoya, M. Ishibashi and K. Koike, Appl. Phys. Lett., 1997, 71, 2934-2936.

19 A. S. Dimitrov and K. Nagayama, Chem. Phys. Lett., 1995, 243, $462-$ 468.

20 G. Y. Onoda and E. G. Liniger, Phys. Rev. A: At., Mol., Opt. Phys., 1986, 33, 715-716.

21 A. Mihi, M. Ocana and H. Miguez, Adv. Mater., 2006, 18, 2244 2249.

22 A. S. Dimitrov and K. Nagayama, Langmuir, 1996, 12, 1303-1311.

23 M. Trau, D. A. Saville and I. A. Aksay, Science, 1996, 272, 706-709.

24 R. C. Hayward, D. A. Saville and I. A. Aksay, Nature, 2000, 404, 5659.

25 S. H. Park, D. Qin and Y. Xia, Adv. Mater., 1998, 10, 1028-1032.

26 Thermo Scientific, NIST Traceable Particle Size Standards, http:// www.thermo.com/eThermo/CMA/PDFs/Various/File_4361.pdf.

27 W. C. Chueh and S. M. Haile, Phys. Chem. Chem. Phys., 2009, 11, 8144-8148. 